International Journal of Engineering \& Technology, $7(3.33)(2018) 33-36$
International Journal of Engineering \& Technology
SPC
Website: $w$ ww.sciencepubco.com/index.php/IJET
Research paper

\title{
Implementation of Facial Visualization of MBTI Personality Type Based on Augmented Reality and Dynamic Mapping
}

\author{
Kang-Hee Lee ${ }^{1 *}$, Asilbek Ganiev ${ }^{2}$ \\ ${ }^{1}$ Department of Digital Media, Soongsil University, Seoul, Republic of Korea, 06978 \\ ${ }^{2}$ Department of Digital Media, Soongsil University, Seoul, Republic of Korea, 06978 \\ *Corresponding author E-mail:kanghee.lee@ssu.ac.kr
}

\begin{abstract}
Nowadays, augmented reality is becoming more and more popular because it can help individuals learn about many things, such as buildings, books, historical places, etc. in real time. The Myers-Briggs Type Indicator (MBTI) test, a questionnaire-based test, is used to determine personality type, which influences perceptions of the world and the ways in which individuals make decisions. This system is based on C.G Jung's typological theory and was developed by Katherine and Isabel Briggs. The purpose of this paper is to use the MBTI personality test to define the personality types of users. To accomplish this aim, we used Unity3D, Processing, and Vuforia SDK. First, the processing program was used to produce results from sixteen images and send them to Unity3D. Second, Vuforia SDK was used to recognize masks in real time and map images to the masks. Then, Unity3D displayed the final mapping result.
\end{abstract}

Keywords: Augmented Reality; Recognizing Target; Mapping Image; Unity3D; Vuforia.

\section{Introduction}

The purpose of this paper is to reveal the types and characteristics of people in images. For a clear understanding of the results, we used pictures of famous people. In this project, we used the MBTI questionnaire, which is based on C.G Jung's typological theory and is designed to help understand people's thoughts, feelings, sensations, and intuition [1]. It was developed by Katherine and Isabel Briggs. We also used augmented reality, which is designed to obtain additional visual information about the real world in real time using a camera. To recognize image targets and to map images, we used Unity3D environment and Vuforia SDK.

In our project, we used MBTI testing to identify personality traits of users, then these results were transformed into sixteen images using a processing program. The processing program sends the images to Unity, which used a camera to recognize image targets and map the resulting images. The structure of this paper is outlined as follows: the next section introduces the software which we used in our project. In Section 3, we describe the architectural design of the whole process. Section 4 illustrates the results of our experiments. Concluding remarks are followed in Section 5.

\section{Development Environment and Back- ground}

\subsection{Unity3D}

Unity3D is multi-platform game engine used to create $2 \mathrm{D}$ and $3 \mathrm{D}$ imaging features, such as animations, physics, objects, and audio and video games.

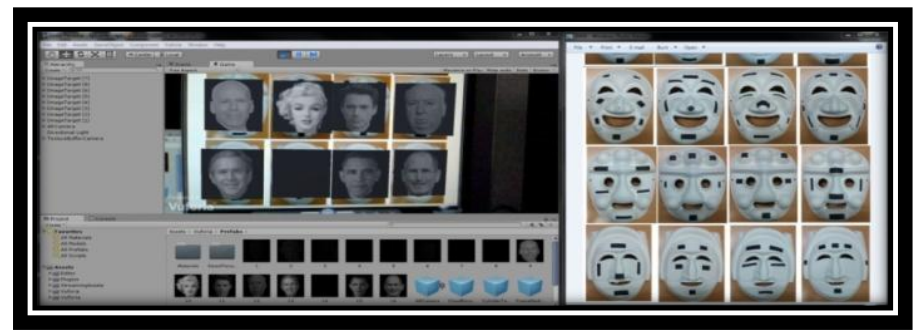

Fig. 1: Unity3D Environment and the proposed marker-masks

Fig. 1. Unity3D Environment and the proposed marker-masks Unity3D has a simple and easily understandable interface for users and is relatively easy to script with C\# or JavaScript. Unity3D is available on the Mac OS X and Windows 7, 8, and 10 operating systems. Developed applications can be run on An-droid, Windows, iOS, WebGL, Windows Phone, PS4, etc. devices [2]. In this project, we used Unity3D with a web camera to recognize and map images in real time.

\subsection{Processing}

Processing is an open source programming language with an easy to use and simple programming environment.

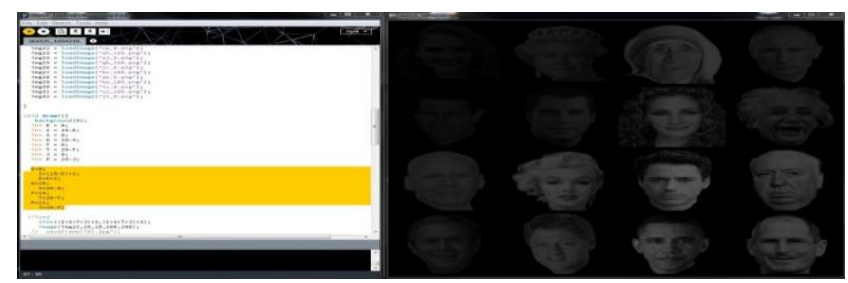

Fig. 2: processing environment and the proposed MBTIi-celebrities 
For users, it makes it easier to develop visually-oriented applications with images, animations, and complex data visualization [3][4]. The processing language builds on the Java programming language, but in comparison with Java it uses a simplified syntax. It is available for the Windows, Mac OS X, and Linux operating systems. In this project, we used processing to obtain 16 images and send them to Unity.

\subsection{Vuforia}

Vuforia is an augmented reality Software Development Kit (SDK) developed by Qualcomm Tech-nologies, which enables the creation of augmented reality applications. Vuforia uses computer vision technology to recognize and track 2D image targets and 3D objects such as boxes, cans, and masks in real time. Vuforia SDK can be run on Android, Windows, and iOS platforms. In order to build applications, it can be used with Xcode, Android Studio, and Unity game engine. In addition, Vuforia has an object scanner application that enables visualization of $3 \mathrm{D}$ objects in a target format [5][6]. In our project, we used Vuforia integrated with Unity to create and track 2D and 3D image targets and Vuforia Object Scanner for 3D scanning masks.

\subsection{Myers-Briggs Type Indicator}

Myers-Briggs Type Indicator (MBTI) is an instrument for measuring a person's preferences, using four basic indices with opposite poles [7][8]. The four indices are: (1) Extraversion/Introversion, (2) Sensate/iNtutive, (3) Thinking/Feeling, and (4) Judging/Perceiving. The MBTI instrument is based on C.G. Jung's ideas about perception and judgement. and the attitudes in which these are used in different types of people [9]. The aim of the MBTI instrument is to identify, from self-report of easily recognized reactions, the basic preferences of people in regard to perception and judgement, so that the effects of each preference, singly and in combination, can be established by research and pit into practical use. According to the Center for Applications of Psychological Type, the MBTI is "the most widely used personality inventory in history" and approximately 2,000,000 people a year take the MBTI. Using these MBTI theory and AR technique, we implement a facial visualization method to visualize various MBTI personality types well. For example, Lee proposed the customized personality generation skill based on MBTI concepts [10]. If he employs the proposed method, his method would be enhanced.

\section{Design of Proposed Architecture}

Figure 3 shows a block diagram outlining our project. In the first block, an MBTI test is performed to determine users' personality traits. The second block shows the translation of the MBTI test results into sixteen different contrast images. This block is composed of making backgrounds to represent traits of each MBTI category using 'Big Five Personality Traits' and showing facial images of celebrities with corresponding MBTI personality types. First, 'Big Five Personality Traits' classifies 16 MBTI types into four categories and each of category traits lays the foundation to make background using visual elements such as colors and lines. Second, celebrities' facial images are substituted for the corresponding literal explanation of each MBTI type [11]. The last block shows the use of Unity3D and Vuforia SDK to recognize targets in real time using a camera and mapping to image targets, as shown in Figure 4.

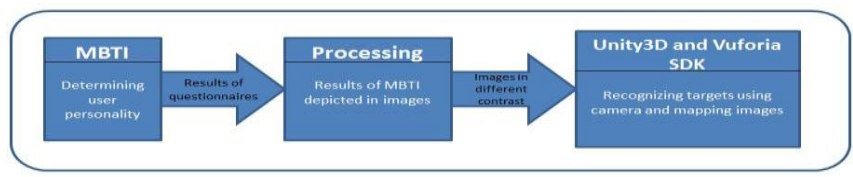

Fig. 3: Block diagram of the proposed architecture

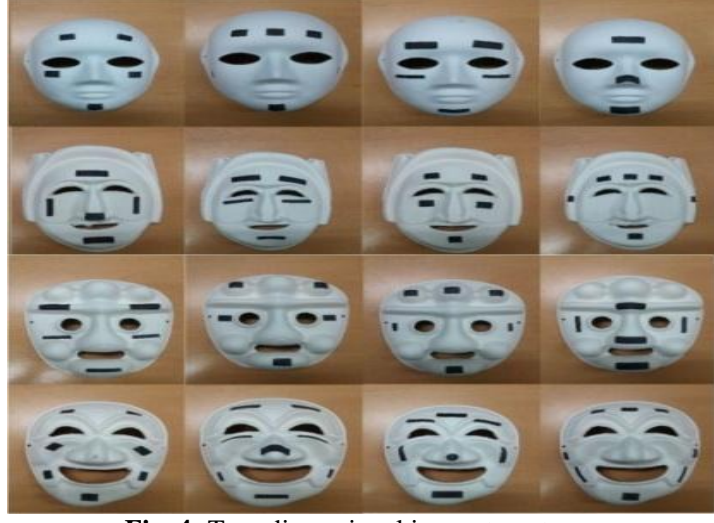

Fig. 4: Two-dimensional image targets

Figure 4 shows four types and sixteen different 2-dimensional images of mask targets. These images were mapped onto images of famous people in Unity 3D.

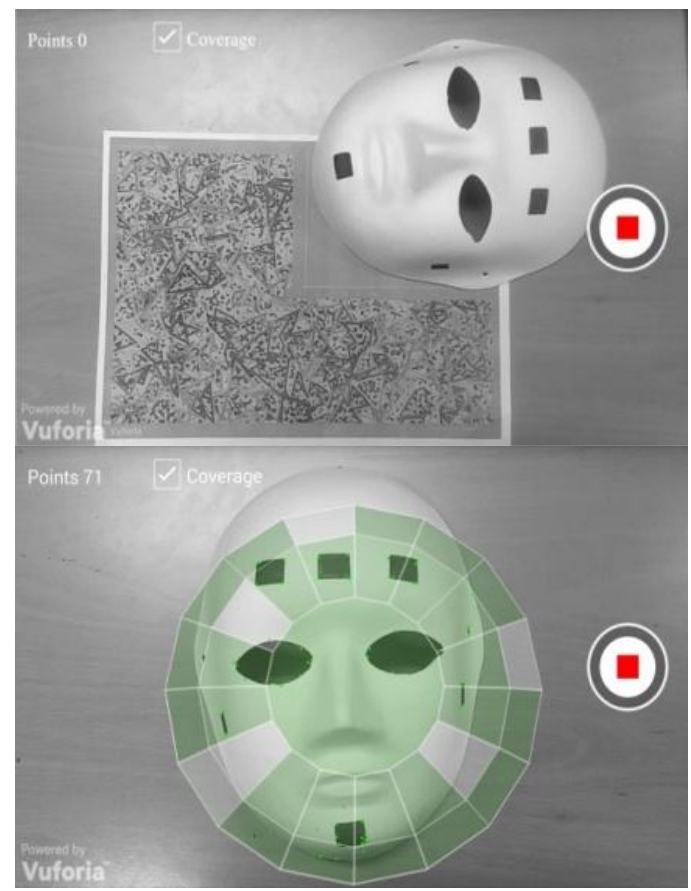

Fig. 5: Three-Dimensional object targets: scanned object(top) and target object with 71 points (bottom)

Figure 5 demonstrates three-dimensional object targets which were created using the Vuforia object scanner in an Android device. These object targets have also been mapped to images in experiments.

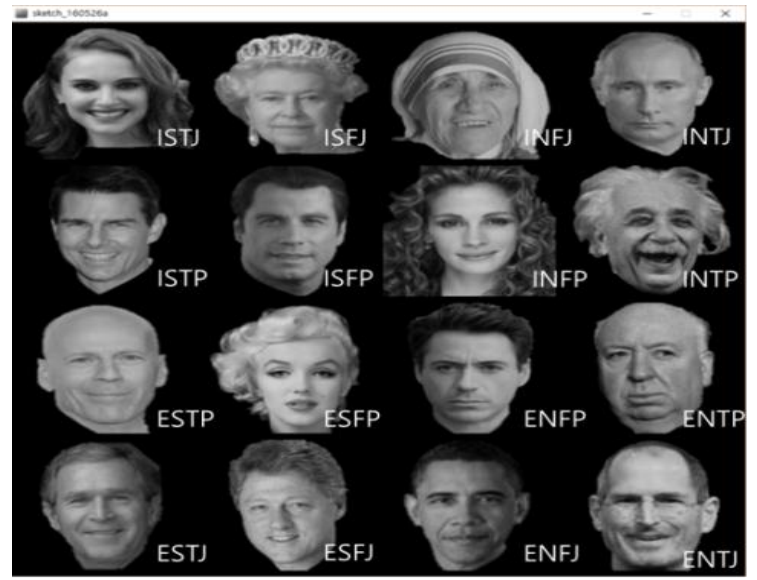

Fig. 6: Images of famous people with their MBTI personality types 
Figure 6 shows sixteen images of famous people, such as presidents, actors, scientists, etc., along with their personality types. These images were used to present the results of MBTI testing and were mapped to masks in Unity3D.

Table 1: Eight characteristics used for MBTI assessment

\begin{tabular}{|c|c|}
\hline (E)-Extraversion & (I)-Introversion \\
\hline (S)-Sensing & (N)-Intuition \\
\hline (T)-Thinking & (F)-Feeling \\
\hline (J)-Judging & (P)-Perceiving \\
\hline
\end{tabular}

Table 1 shows the eight characteristics used in MBTI assessment. By combining these eight characteristics, MBTI produces sixteen possible personality types as shown in table 2 .

Table 2: Sixteen possible MBTI Personality type(Lower)

Table 2: Sixteen possible MBTI Personality type(Lower)
\begin{tabular}{|c|c|c|c|}
\hline ISTJ & ISFJ & INFJ & INTJ \\
\hline ISTP & ISFP & INFP & INTP \\
\hline ESTP & ESFP & ENFP & ENTP \\
\hline ESTJ & ESFJ & ENFJ & ENTJ \\
\hline
\end{tabular}

Between EI, Extraversion or Introversion affects choices as to whether to direct perception judgment mainly on the outer world (E) or mainly on the inner world of ideas. Between SM, Sensing perception or iNtutive perception affects choices as to which kind of perception is preferred when one needs or wishes to perceive. Between TF, Thinking judgment or Feeling judgment affects choices as to which kind of judgment to trust when one needs or wishes to make a decision. Between JP, Judgment or Perception affects choices as to whether to deal with the outer world in judging (J) attitude (using T or F) or in the perceptive (P) attitude (using $\mathrm{S}$ or $\mathrm{N}$ ).

\section{Experiments}

This section details the experimental results of our project. In our experiments, we used MBTI testing to determine personality type, which reflects how individuals perceive the world and make decisions. To do so, we used questionnaires, processing to demonstrate MBTI results in images, and Unity3D and Vuforia SDK to recognize targets and map the images to masks. In addition, using Unity, we created an application for Android devices which was also used in our experiments.

\subsection{Experiment 1: Personality Visualization through PC}

Figure 7 shows the experimental results from image processing. Processing was used to demonstrate the results of MBTI in images. The resultant images have different levels of contrast depending on users' results. For example, if a resulting image shows a high (dark) level of contrast, this means that the user has dissimilar personality traits to those of the person shown in the image; on the other hand, if an obtained image has low (light) contrast, this indicates that the user has a high degree of similarity with the characteristics of the famous person in the image.

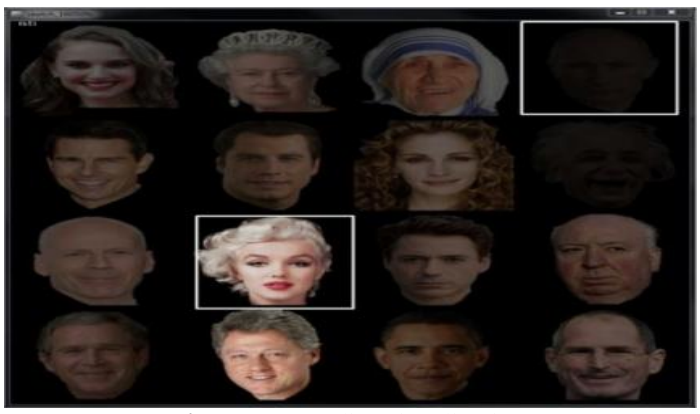

Fig. 7: Personality visualization
Experiment1 demonstrate results, which illustrate dominant and inferior figures. The inferior result in this case is Vladimir Putin, who has the INTJ (Introversion, iNtuition with Thinking and Judging) personality type, whereas the dominant result is Marilyn Monroe, who has the ESFP (Extraversion, Sensing with Feeling and Perceiving) personality type, which is associated with:

A. Feeling motivated by interactions with other people

B. Being more concrete than abstract

C. Valuing personal considerations above objective criteria

D. Withholding judgment and delaying important decisions

E. A strong ability to understand the objective world

F. Being skilled at finding the best things for themselves and others.

\subsection{Experiment 2: Personality Visualization through Static Mapping}

In this project, Unity3D and Vuforia SDK were used to recognize images and target objects. Unity uses a camera to recognize targets in real time. Also, Unity uses data from image processing, which sends sixteen images to the Unity folder.

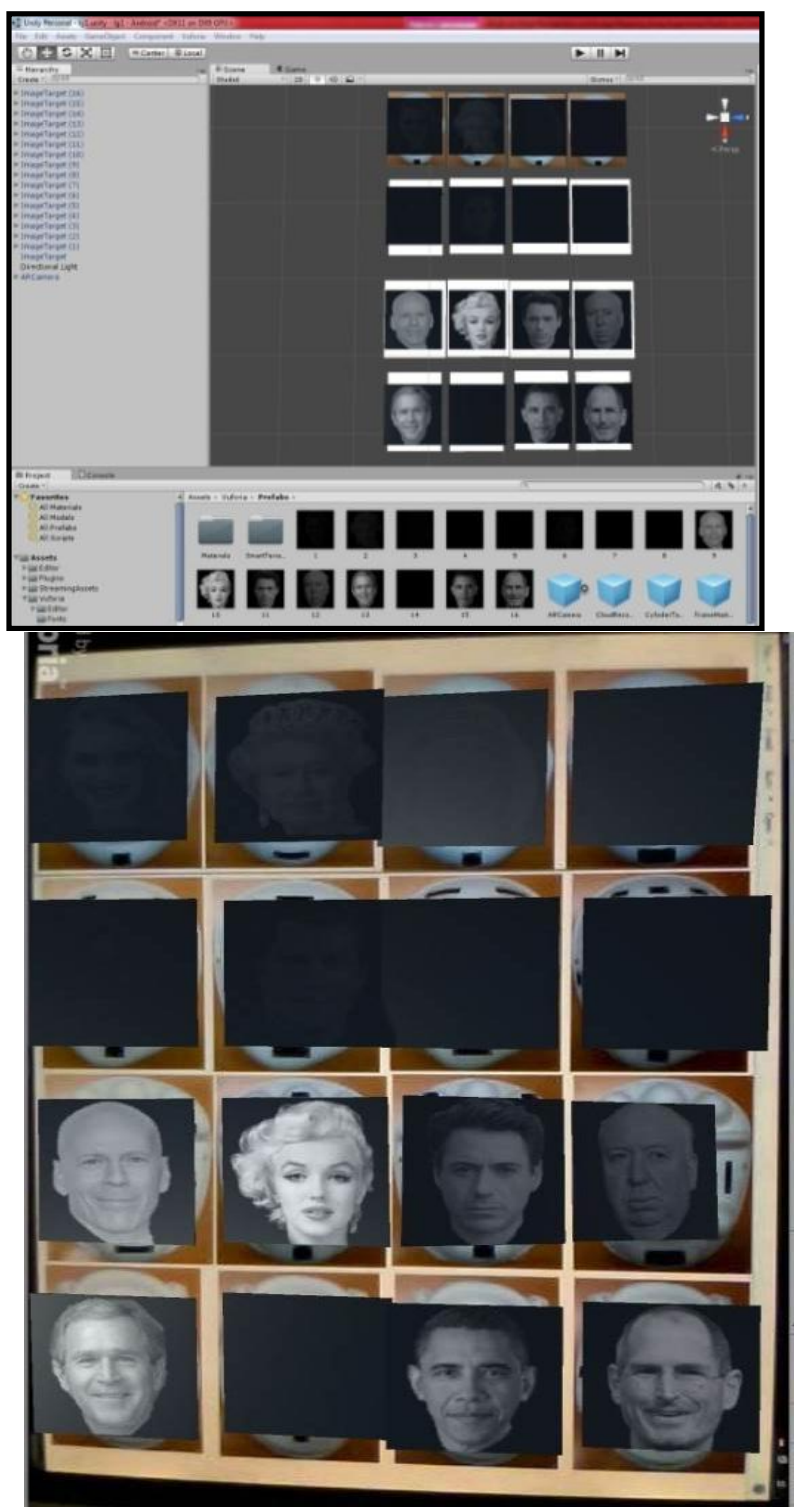

Fig. 8: Static mapping: unity environment (top) and mapping in real time (bottom)

Figure 8 shows the Unity3D environment with image targets and images produced by processing and images mapped to targets in real time. 


\subsection{Experiment 3: Personality Visualization through Dynamic Mapping}
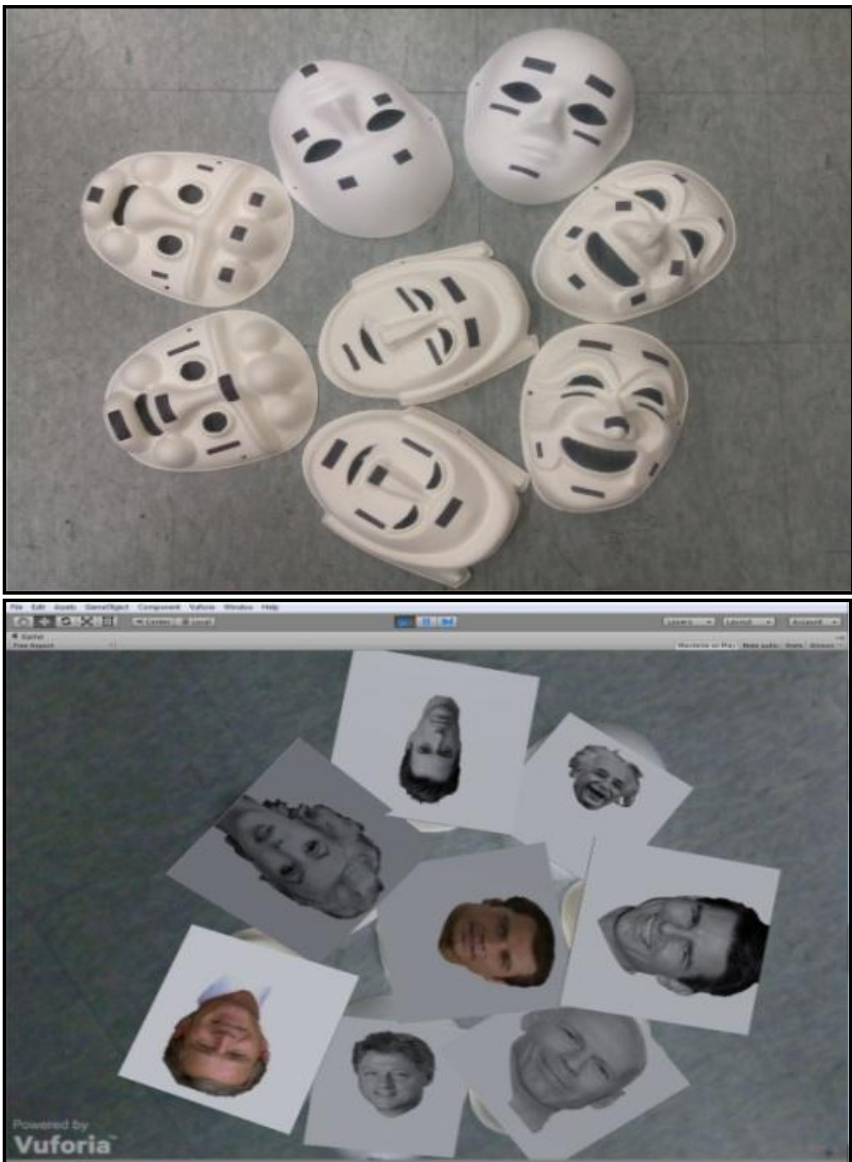

Fig. 9: Dynamic mapping: random image targets (top) and randomly mapped images (bottom)

Figure 9 shows an experimental result for randomly mapped images in real time for real masks. In this experiment, we used eight different masks to randomly map images. The advantages of this experiment are that masks can be moved to any location in view of the camera and can be rotated 360degree.

\subsection{Experiment 4: Integrated Personality Visualization through a Smartphone}

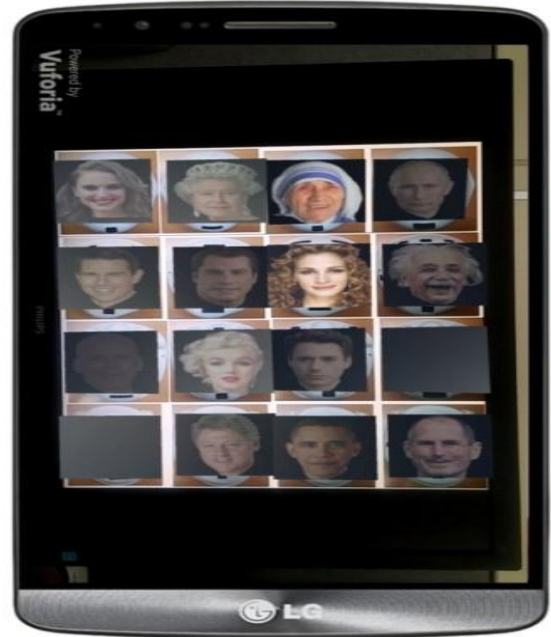

Fig. 10: Integrated Personality Visualization: target recognition and map ping of images using an android
Because we used the Android platform, our application works on smartphones and other Android devices. Figure 10 shows the results of target recognition and mapping of images, similar to experiment 2 which is shown in Figure 8 . The advantage of this experiment is that it can be used with $3 \mathrm{D}$ glasses in smartphones, so the results will be more realistic.

\section{Conclusions}

The main purpose of this project was to define the characteristics and personality types of users by comparison with images of famous people, and to map them onto image targets. The personality types of the users were determined with MBTI testing, which makes it possible to produce clear and useful data. The results from MBTI testing were transformed into images using a processing program with images of sixteen different famous people at different levels of contrast, which indicated how similar or dissimilar a user's personality type and characteristics are with the resultant images. To display the results, we used Unity3D and Vuforia SDK, which allow for image mapping in real time. This project used different methods, such as mapping and construction of sixteen images using a PC and web camera. Another experiment involved random mapping onto image targets where the targets were located in a random position and at different angles. Images were dynamically mapped with targets located at different distances from the camera. In addition, using Unity3D, we created an Android application for the project which works in smartphones and other Android-based platforms. The advantage of the application is that it produces better and faster results of target recognition in real time.

\section{Acknowledgement}

This research was supported by Basic Science Research Program through the National Research Foundation of Korea(NRF) funded by the Ministry of Education(NRF-2017R1D1A1B05036028).

\section{References}

[1] C.G.Jung, Psychologische Typologie, Süddeutsche Monatshefte., Vol 33, (1936), pp. 264-272.

[2] M. Patrick, T. Marcus, K, Gudron. Augmented Reality for teaching spatial relations, Conference of the International Journal of Arts \& Sciences, (2009), Vol 2.

[3] Unity 3D, available online: https://unity3d.com/

[4] Processing available at, Retrieved from https://processing.org/

[5] Fry and Ben, Visualizing Data (1st ed.), O'Reilly Media, ISBN 0596-51455-7, Vol. 19, (2008).

[6] Developing with Vuforia, available online: https://developer.vuforia.com/library/getting-started/

[7] MBTI Foundation. Personality types of the MBTI, available online: http://www.myersbriggs.org/my-mbti-personality-type/mbtibasics/the-16-mbti-types.htm

[8] Isabel Briggs Myers, Perter B. Myers, Gifts Differing - Understanding Personality Type, Davies-Black Publishing, Mountain View, California, (2010)

[9] Carl Jung, Psychological Types, Taylor \& Francis Group, (2016).

[10] K.H. Lee, Y. Choi, and D. J. Stonier, Evolutionary algorithm for a genetic robot's personality based on the Myers-Briggs Type Indicator, Robotics and Autonomous Systems, Vol. 60, (2012), pp. 941961.

[11] Ho-Sun shin and Kang-Hee Lee, A proposal of visualization method of MBTI personality types using celerity images, Asia-pacific Journal of Multimedia Services Convergent with Art, Humanities, and Sociology, Vol. 6, No., 8, (2016), pp. 491-498. 